\title{
Determinants of recurrent atrial flutter after cardioversion
}

\author{
RICHARD G POZEN, JORGE PASTORIZA, JOHN J ROZANSKI, KENNETH M KESSLER, \\ ROBERT J MYERBURG \\ From the Division of Cardiology, Department of Medicine, University of Miami School of Medicine, Miami, Florida, \\ USA
}

SUMMARY Eighteen male patients (mean age 59 years) who were electrically cardioverted for pure atrial flutter were retrospectively studied to determine those factors influencing the maintenance of regular sinus rhythm or reversion to atrial flutter. Six months after successful cardioversion, 10 patients $(55 \%)$ had recurrent atrial flutter and eight patients $(45 \%)$ were still in sinus rhythm. The two groups were not significantly different with respect to age, symptomatology, abnormalities on the 12 lead electrocardiogram (during sinus rhythm), or the administration of digoxin and a class Ia antiarrhythmic agent (after cardioversion). There was a trend for those patients with recurrent atrial flutter to have a higher incidence of underlying heart disease and previous episodes of atrial flutter than the non-recurrent group. There were statistically significant differences between the recurrent and non-recurrent groups with respect to echocardiographically determined left atrial size and left ventricular ejection fraction. Patients with a left atrial size greater than $45 \mathrm{~mm}$ or with an ejection fraction less than $45 \%$ were all at high risk for recurrent atrial flutter after successful cardioversion.

Previous studies in patients with atrial fibrillation have shown a strong correlation between failure to maintain sinus rhythm after cardioversion of this arrhyth$\mathrm{mia}$ and the presence of underlying heart disease, an enlarged left atrium on echocardiogram or chest $x$-ray film, a history of previous episodes of atrial fibrillation, older age patients, the duration of atrial fibrillation before cardioversion, and the use of antiarrhythmic agents. ${ }^{1-9}$ The correlation between atrial ftutter and these factors has not been studied. Electrophysiologically, there are significant differences between atrial fibrillation and atrial flutter. ${ }^{19}$ More importantly, these two arrhythmias differ in terms of their clinical behaviour with respect to incidence, patients at risk, ease of cardioversion, duration of the arrhythmia, and relapse rate..$^{1-35}$ Previous studies have only included a few patients with atrial flutter and no mention is made of whether they had atrial flutter exclusively or also had documented episodes of atrial fibrillation.

The purpose of this study was to review the outcome of electrical cardioversion in patients with pure atrial flutter, and to determine those factors influencing the maintenance of regular sinus rhythm or reversion to atrial flutter after successful cardioversion.

\footnotetext{
Supported in part by an NIH grant.
}

Accepted for publication 8 February 1983

\section{Patients and methods}

The study population consisted of 18 male patients who were electrically cardioverted for atrial flutter in the coronary care unit of the Miami Veterans Administration Medical Center between 1977 and 1980 and in whom adequate echocardiograms were obtained within two weeks before cardioversion. Patients with atrial fibrillation or flutter fibrillation and postoperative patients were not included. The clinical records of each were retrospectively reviewed to determine the patient's age at the time of cardioversion, the presence or absence of underlying heart disease, the symptoms at the time of admission to hospital, the drugs given after cardioversion, and subsequent clinical course and electrocardiographic findings.

\section{ELECTROCARDIOGRAPHIC EVALUATION}

The diagnosis of atrial flutter was determined according to conventional criteria. ${ }^{10}$ Electrocardiograms and clinical records were reviewed for the presence of previous episodes of atrial flutter or atrial fibrillation. Patients with a history or documentation of atrial fibrillation at any time were excluded. After cardioversion all patients were followed for at least six months unless atrial flutter recurred earlier. Electrocardiograms, after cardioversion, during regular 
sinus rhythm were interpreted for the presence of left atrial abnormality ( $p$ wave duration $\geqslant 0.12 \mathrm{~s}$ in standard lead or terminal negative component $\geqslant 0.04$ $\mathrm{mm} \mathrm{s}$ in V1), left ventricular hypertrophy, first degree atrioventricular block, intraventricular conduction disturbances, non-specific ST-T wave changes, and a pattern of previous myocardial infarction.

\section{ECHOCARDIOGRAPHIC EVALUATION}

All patients had an M-mode echocardiogram performed within the two weeks before cardioversion. Echocardiograms were performed with the patient either in the supine or left lateral decubitus position using an Irex 101, Picker $80 \mathrm{C}$, or Unirad C system interfaced with a Honeywell strip chart recorder. Echocardiograms were assessed for left atrial and left ventricular dimensions according to the recommendations of the committee on M-mode standardisation of the American Society of Echocardiography. ${ }^{11}$ Left ventricular ejection fraction was calculated by the Teichholz method. ${ }^{12}$

\section{STATISTICS}

Because of the skewed distribution of the echocardiographic and chronological variables, median differences between the recurrent atrial flutter group and the non-recurrent group were analysed by the Wilcoxon two sample rank tests and $\chi^{2}$ analysis. Data for the entire group are expressed as mean \pm standard deviation.

\section{Results}

\section{CLINICAL DATA}

There were 18 male patients with a mean age of 59 years (range 49 to 71 ) at the time of cardioversion. Twelve of the patients $(67 \%)$ had evidence of underlying heart disease, including mitral valve disease (three patients), aortic valve disease (two patients), atherosclerotic heart disease (four patients), and cardiomyopathy (three patients). Six patients (33\%) had no evidence of underlying heart disease by history, physical examination, chest $x$-ray examination, electrocardiogram, or echocardiogram. Thirteen patients (72\%) were symptomatic at the time of admission and five patients $(28 \%)$ were asymptomatic. Symptoms included dyspnoea (10 patients), palpitation (eight patients), angina pectoris (two patients), and stroke (one patient).

Electrical cardioversion was performed without complications in all patients. Fourteen patients $(78 \%)$ were given digoxin and a type IA antiarrhythmic agent (quinidine, procainamide, or disopyramide) after cardioversion. Four patients received digoxin alone or no drugs.

\section{ELECTROCARDIOGRAPHIC OBSERVATIONS}

All patients had sustained atrial flutter of the common type (typical saw-tooth baseline with superior orientation of the electrical axis of the flutter waves) with atrial rates varying from 250 to $350 / \mathrm{min}$ and ventricular responses from 75 to $160 / \mathrm{min}$. Eight patients (44\%) had at least one previously documented episode of atrial flutter. During regular sinus rhythm after cardioversion, the 12 lead electrocardiogram showed the following abnormalities: left atrial abnormality (44\%), left ventricular hypertrophy (39\%), first degree atrioventricular block (22\%), intraventricular conduction disturbance (28\%), myocardial infarction (17\%), and non-specific ST-T wave changes (78\%).

All 18 patients were successfully cardioverted to regular sinus rhythm which persisted from one day to five years. Six patients (33\%) reverted to atrial flutter within the first month and an additional four patients reverted within six months. The remaining eight patients (45\%) maintained regular sinus rhythm for at least six months after cardioversion.

\section{ECHOCARDIOGRAPHIC RESULTS}

Mean left atrial size for all 18 patients was $43 \cdot 1 \pm 5 \cdot 8$ $\mathrm{mm}$ (range 36 to $53 \mathrm{~mm}$ ). Seven patients (38\%) had a left atrial size of $40 \mathrm{~mm}$ or less, five patients (28\%) had a left atrial diameter of 41 to $45 \mathrm{~mm}$, and six patients (33\%) had a left atrium larger than $45 \mathrm{~mm}$. Mean left ventricular end-diastolic dimension (LVEDD) was $53.1 \pm 10.0 \mathrm{~mm}$ (range 36 to 84 ). Twelve patients $(67 \%)$ had a left ventricular size of $55 \mathrm{~mm}$ or less and six patients $(33 \%)$ had left ventricular dilatation (LVEDD greater than $55 \mathrm{~mm}$ ). Four of the six patients $(67 \%)$ with a dilated left ventricle also had a left atrium greater than $45 \mathrm{~mm}$, whereas only two of 12 patients $(17 \%)$ with a normal sized left ventricle had an enlarged left atrium $(p<0.05)$.

Left ventricular ejection fraction by echocardiogram ranged from 20 to $95 \%$ (mean $52 \cdot 6 \pm 20.4 \%$ ). Ejection fraction was less than $45 \%$ in seven patients (39\%) and was $45 \%$ or greater in 11 patients $(61 \%)$. There was no statistical correlation between left ventricular ejection fraction and left atrial size or left ventricular size by echocardiography or between ejection fraction and left ventricular hypertrophy by electrocardiogram.

\section{DETERMINANTS OF RECURRENT ATRIAL \\ FLUTTER}

There were no statistically significant differences between patients who maintained sinus rhythm and those who reverted to atrial flutter with respect to age, symptoms at the time of admission, or the administration of digoxin and antiarrhythmic agents after cardioversion. Seven of the 10 patients $(70 \%)$ with recurrent atrial flutter and five of the eight patients (62\%) 
without recurrence had evidence of underlying heart disease. There was a trend for the six patients with recurrent atrial flutter at one month after cardioversion to have a higher incidence of underlying heart disease compared with the remaining 12 patients (five of six $(83 \%)$ versus seven of $12(59 \%)$, respectively). There was also a trend for the eight patients with a previous history of atrial flutter to have recurrent atrial flutter compared with the 10 patients in whom this was their initial episode (six of eight (75\%) versus four of 10 (40\%), respectively).

There were no electrocardiographic variables predictive of recurrent atrial flutter. Neither left atrial abnormality (four of $10(40 \%)$ with, and four of eight (50\%) without recurrent atrial flutter showed left atrial abnormality), left ventricular hypertrophy (three of $10(30 \%)$ with and four of eight $(50 \%)$ without recurrent atrial flutter had left ventricular hypertrophy), atrial rate during flutter, nor the presence of first degree atrioventricular block, intraventricular conduction disturbances, non-specific ST-T wave abnormalities, or myocardial infarction were predictive of recurrence. Investigation of a possible relation between recurrence and ST-T abnormalities is confounded by the fact that post-tachycardiac ST-T abnormalities could not be discerned from chronic ST-T abnormalities in every patient.

In contrast to left atrial enlargement by electrocardiography, median left atrial size was significantly larger by echocardiography in the 10 patients with recurrent atrial flutter compared with the eight patients who remained in sinus rhythm (48.5 versus $39.5 \mathrm{~mm}, \mathrm{p}<0.05)$. Only four of the 12 patients (33\%) with a left atrial size less than $45 \mathrm{~mm}$ had recurrent atrial flutter, whereas all six patients with a left atrium greater than $45 \mathrm{~mm}$ had recurrent atrial flutter $(\mathbf{p}<0.01)$ (Fig.).

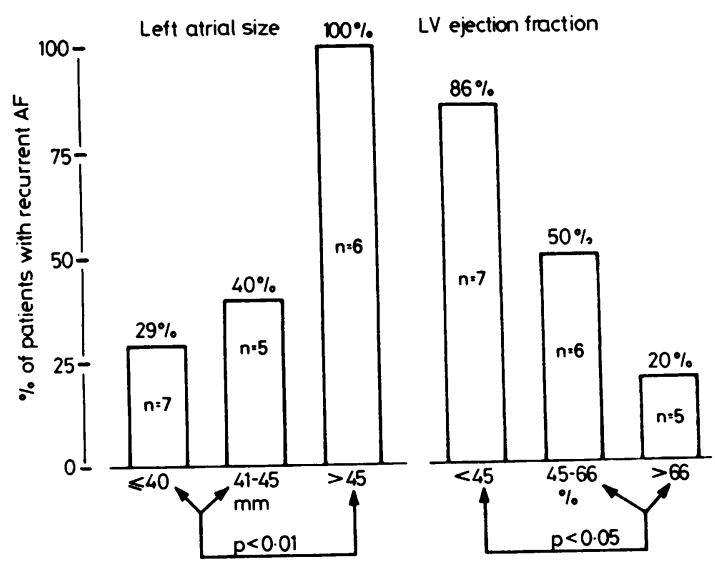

Fig. Frequency of recurrent atrial ftutter $(A F)$ according to left atrial size and lefi ventricular $(L V)$ ejection fraction.
Left ventricular ejection fraction was also a good predictor of recurrent atrial flutter. Six of seven patients (86\%) with an ejection fraction less than $45 \%$ had recurrent atrial flutter compared with four of 11 patients $(36 \%)$ with an ejection fraction greater than $45 \%(p<0.05)$ (Fig.). The median ejection fraction for the group with recurrent atrial flutter was $37.5 \%$ compared with $63.5 \%$ in the group without recurrent atrial flutter $(p=0.05)$. In contrast, left ventricular end-diastolic dimension was not a good predictor of recurrent atrial flutter. Six of the 12 patients (50\%) with a left ventricular end-diastolic dimension less than $55 \mathrm{~mm}$ and four of the six patients $(67 \%)$ with a left ventricular end-diastolic dimension greater than $55 \mathrm{~mm}$ had recurrent atrial flutter $(p=N S)$.

\section{Discussion}

Patients successfully cardioverted from atrial fibrillation to regular sinus rhythm have a variable rate of recurrence of atrial fibrillation. Previous investigations have shown that there are certain clinical, radiological, and echocardiographic characteristics which may help identify those patients at highest risk for recurrence. The present study retrospectively examined these and other variables in a group of 18 patients with pure atrial flutter who underwent successful electrical cardioversion.

Several studies have shown that the duration of atrial fibrillation is an important predictor of both the success of cardioversion and the ability to maintain regular sinus rhythm after cardioversion. ${ }^{1-6}$ The present study could not address this because atrial flutter was treated when first noted and the true duration could not be determined. Our data showed, however, a trend for patients with previous episodes of atrial flutter to be at higher risk for recurrence compared with patients with an initial episode of atrial flutter, as has been previously shown for patients in atrial fibrillation. ${ }^{135}$

In atrial fibrillation, the presence of underlying heart disease is also significantly associated with a higher rate of recurrence after successful cardiover- No sion. ${ }^{2-57}$ Our data for patients with atrial flutter are also in agreement with previous reports, especially at a follow-up of one month. Only one of six patients (17\%) without underlying heart disease had recurrent atrial flutter, whereas five of 12 patients (42\%) with underlying heart disease had recurrent atrial flutter at one month after cardioversion. The number of patients with specific cardiac lesions, however, was too small to examine each pathological entity separately.

It is still controversial in patients with atrial fibrillatin whether class Ia antiarrhythmic drugs decrease the incidence of recurrent atrial fibrillation..$^{1-313-15}$ The 
present study does not show any advantage for using digoxin and a class Ia antiarrhythmic agent in patients with atrial flutter who have been cardioverted. This study, however, was neither randomised nor were drug doses or serum levels uniform. Furthermore, too few patients were analysed to consider each class Ia antiarrhythmic separately. Future prospective studies monitoring drug dosing and blood levels must be obtained in order to establish whether or not class Ia antiarrhythmics are valuable after cardioversion in the treatment of pure atrial flutter.

As opposed to echocardiography, the standard 12 lead electrocardiogram was not helpful in identifying patients likely to have recurrent atrial flutter. Inexact correlation between echocardiographic and electrocardiographic findings may be one reason for this discrepancy. On further analysis of our data we incidentally found that the sensitivity, specificity, and predictive accuracy of left atrial abnormality on the routine electrocardiogram compared with left atrial size on echocardiogram were only $25 \%, 60 \%$, and $33 \%$, respectively. The presence of left ventricular hypertrophy on the routine electrocardiogram, however, was $83 \%$ sensitive and specific and had a predictive accuracy of $71 \%$ for detecting a left ventricular end-diastolic dimension greater than $55 \mathrm{~mm}$ by echocardiogram.

The echocardiogram, as in patients with atrial fibrillation, was the best technique for predicting the recurrence of atrial flutter. The group of patients with recurrent atrial flutter had a significantly larger median left atrial size than those who maintained sinus rhythm for at least six months (48.5 versus 39.5 $\mathrm{mm}, \mathrm{p}<0.05$ ). The sensitivity, specificity, and predictive value of a left atrial diameter greater than $45 \mathrm{~mm}$ for the probability of recurrent atrial flutter were $60 \%, 100 \%$, and $100 \%$, respectively. These data are in agreement with those of Ewy et al. ${ }^{7}$ and Henry et al. ${ }^{6}$ who measured left atrial size echocardiographically in patients with atrial fibrillation. We also found that there was a significant association between left atrial enlargement and left ventricular enlargement.

Finally, our data demonstrate that those patients with abnormal left ventricular function (ejection fraction less than $45 \%$ ) are also at significantly greater risk for recurrent atrial flutter. The sensitivity, specificity, and predictive value of an ejection fraction less than $45 \%$ for predicting recurrence of atrial flutter after successful cardioversion were $60 \%, 87 \%$, and $86 \%$, respectively. This association between a decreased ejection fraction and recurrence of arrhythmias has not previously been described.

In summary, in patients with atrial flutter (as reflected by this study of 49 to 71 year old male veterans) the ability to maintain regular sinus rhythm for at least six months after successful cardioversion can be predicted by left atrial size and left ventricular ejection fraction as determined by $\mathrm{M}$-mode echocardiography. Helpful, though not as predictive, are a history of previous episodes of atrial flutter or the presence of significant underlying heart disease. Of little or no value in distinguishing patients at high risk for recurrent atrial flutter are the standard 12 lead electrocardiogram, symptomatology, age, or the administration of digoxin and class Ia antiarrhythmic medication.

\section{References}

1 Morris JJ Jr, Kong Y, North WC, McIntosh HD. Experience with "cardioversion" of atrial fibrillation and flutter. Am f Cardiol 1964; 14: 94-100.

2 Razavi M, Duarte EP, Tahmooressi P. Cardioversion: ten-year Cleveland clinical experience. Clev Clin Q 1976; 43: 175-80.

3 Resnekov L, McDonald L. Appraisal of electroconversion in treatment of cardiac dysrhythmias. $\mathrm{Br}$ Heart $\mathrm{f}$ 1968; 30: 786-811.

4 McCarthy C, Varghese PJ, Barritt DW. Prognosis of atrial arrhythmias treated by electrical counter shock therapy: a three-year follow up. $B r$ Heart $\mathcal{f}$ 1969; 31: 496-500.

5 Jensen JB, Humphries JO, Kouwenhoven WB, Eng DE, Jude JR. Electroshock for atrial flutter and atrial fibrillation: follow up studies on 50 patients. FAMA 1965; 194: $1181-4$.

6 Henry WL, Morganroth J, Pearlman AS, et al. Relation between echocardiographically determined left atrial size and atrial fibrillation. Circulation 1976; 53: 273-9.

7 Ewy GA, Ulfers L, Hager WD, Rosenfeld AR, Roeske WR, Goldman S. Response of atrial fibrillation to therapy: role of etiology and left atrial diameter. $\mathcal{f}$ Electrocardiol 1980; 13: 119-23.

8 Oram S, Davies JPH. Further experience of electrical conversion of atrial fibrillation to sinus rhythm: analysis of 100 patients. Lancet 1964; i: 1294-8.

9 Waris E, Kreus KE, Salokannel J. Factors influencing persistence of sinus rhythm after DC shock treatment of atrial fibrillation. Acta Med Scand 1971; 189: 161-6.

10 Marriott HJL, Myerburg RJ. Recognition and treatment of cardiac arrhythmias and conduction disturbances. In: Hurst JW, ed. The heart. 4th ed. New York: McGrawHill, 1978: 637-94.

11 Sahn DJ, DeMaria A, Kisslo J, Weyman A. The committee on M-mode standardization of the American Society of Echocardiography: recommendations regarding quantitation in M-mode echocardiography: results of a survey of echocardiographic measurements. Circulation 1978; 58: 1072-83.

12 Teichholz LE, Kreulen T, Herman MV, Gorlin R. Problems in echocardiographic volume determinations: echocardiographic-angiographic correlations in the presence or absence of asynergy. Am $\mathcal{F}$ Cardiol 1976; 37: 7-11.

13 Södermark T, Jonnson B, Olsson A, et al. Effect of quinidine on maintaining sinus rhythm after conversion 
of atrial fibrillation or flutter: a multicentre study from Stockholm. Br Heart f 1975; 37: 486-92.

14 Byrne-Quinn E, Wing AJ. Maintenance of sinus rhythm after DC reversion of atrial fibrillation: a double blind controlled trial of long acting quinidine bisulphate. $\mathrm{Br}$ Heart f 1970; 32: 370-6.

15 Hillestad L, Bjerklund C, Dale J, Maltau J, Storstein O. Quinidine in maintenance of sinus rhythm after elec- troconversion of chronic atrial fibrillation: a controlled clinical study. Br Heart f 1971; 33: 518-21.

Requests for reprints to Dr.Kenneth M Kessler, Division of Cardiology (D-39), Department of Medicine, University of Miami School of Medicine, PO Box 016960, Miami, Florida 33101, USA. 